\title{
Radial flow of kaon mesons in heavy ion reactions
}

\author{
Z.S.Wang, Amand Faessler, C. Fuchs, V.S.Uma Maheswari and T.Waindzoch \\ Institut für Theoretische Physik der Universität Tübingen, \\ Auf der Morgenstelle 14, D-72076 Tübingen, Germany
}

\begin{abstract}
This work investigates the collective motion of kaons in heavy ion reactions at SIS energies (about 1-2 GeV/nucleon). A radial collective flow of $K^{+}$mesons is predicted to exist in central $\mathrm{Au}+\mathrm{Au}$ collisions, which manifests in a characteristic "shoulder-arm" shape of the transverse mass spectrum of the midrapidity $K^{+}$mesons. The $K^{+}$radial flow arises from the repulsive $K^{+}$mean field in nuclear matter. In spite of a strong reabsorption and rescattering the attractive $K^{-}$mean field leads as well to a collective radial flow of $K^{-}$ mesons. The $K^{-}$radial flow, however, is different from that of $K^{+}$mesons and can be observed by a characteristic "concave" structure of the transverse mass spectrum of the $K^{-}$ mesons emitted at midrapidity. The kaon radial flows can therefore serve as a novel tool for the investigation of kaon properties in dense nuclear matter.
\end{abstract}

$25.75 .+\mathrm{r}$ 


\section{INTRODUCTION}

Collective motion in heavy ion reactions has attracted increasing experimental and theoretical interests. "Bounce-off" [1] 3], "squeeze-out" [4, 5] as well as radial flow [6 8 of nuclear matter is predicted by theories and observed in experiments. By "bounce-off" one means a deflection of the spectators sidewards in the reaction plane, while "squeeze-out" denotes nuclear matter jets in the direction perpendicular to the reaction plane emitted from the high density overlap region ( so-called fireball). Radial flow is a collective expansion of the compressed nuclear matter. The collective motion has proved to be a good probe for the equation of state of nuclear matter (EOS) [9,10] as well as for the heavy ion reaction dynamics [11].

The techniques developed in studying the collective flow of nuclear matter have also been

applied to secondary particles such as pions, kaons, hyperons etc. [14 26]. For secondary particles "squeeze-out" and "transverse flow" are defined in the same way as for nucleons by the azimuthal anisotropy and the rapidity dependence of the in-plane component of the particle momentum, respectively. The prediction of a pion transverse flow anticorrelated with that of nucleons has been confirmed by experiments [14 16]. Kaon transverse flow attracts also attention since it might provide information about possible modifications of kaon properties in dense nuclear matter [17 [19]. The in-medium kaon properties are an important issue not only for nuclear physics, but also for astrophysics with respect to the evolution of compact stars [27]. Secondary particles are usually created from elementary hadron-hadron collisions which take place randomly in the colliding system. In the spirit of a proposal by Fermi [28], multiple particle productions from energetic hadron-hadron collisions can be understood in terms of a thermal description. Due to the fairly strong interaction between particles involved in a collision, the available phase space will be occupied according to statistical laws. This physical picture has been supported by extensive experiments on particle production from heavy ion reactions. Therefore, a collective motion of secondary 
particles should be identified by observing deviations from the pure thermal description. It still remains an open question if secondary particles can exhibit collective motion in heavy ion reactions in this strict sense.

In this paper, we explore the possible existence of a collective motion of $K^{+}$and $K^{-}$ mesons in heavy ion reactions by comparing to a pure thermal picture. We mainly consider $\mathrm{Au}+\mathrm{Au}$ reactions at SIS energies ( about 1-2 GeV/nucleon ), since this system has been extensively studied by the experimentalists at GSI/Darmstadt. Secondly, at an incident energy below the the kaon production threshold in free NN collisions ( about $1.58 \mathrm{GeV} /$ nucleon for $K^{+}$mesons and $2.5 \mathrm{GeV} /$ nucleon for $K^{-}$mesons), one can expect a low thermal velocity of the kaons. Consequently, a collective motion, if it exists, will show up clearly. In the present study we are especially interested in kaons emitted at midrapidity. These kaons are most likely produced in compressed nuclear matter stopped in the overlap region of colliding nuclei ( the so-called fireball ). The reaction dynamics is thereby described within the transport approach of Quantum Molecular Dynamics (QMD). We find in this work that both the midrapidity $K^{+}$and $K^{-}$mesons exhibit a novel collective motion in radial direction, which we call kaon radial flow. We demonstrate that the kaon radial flow can provide information on in-medium modifications of kaon properties.

This paper is organized as follows. In Section 2, we briefly sketch the QMD approach and describe the treatment of the kaon dynamics. In Section 3 we elaborate the signature of the $K^{+}$radial flow and demonstrate its collective nature. In Section 4, we study the $K^{-}$ radial flow, which is found to be quite different from the $K^{+}$flow. The role of rescattering and reabsorption of the $K^{-}$mesons is also investigated. In Section 5 we summarize the main results and conclusions of this work.

\section{DESCRIPTION OF KAONS IN QMD}




\section{A. QMD model}

The QMD model [29] has been extensively used for the investigation of heavy ion reactions. In addition to nucleons, we include also $\Delta$ and $N^{*}$ resonances, which turned out to play a non trivial role at an incident energy of 1-2 GeV/nucleon [30,31. Pions are produced by the decay of these resonances. Their Coulomb interaction with the baryons is also included. This is important for a correct description of the pion dynamics, since the pion phase space is highly isospin dependence [22, 32, 33].

\section{B. Sources of $K^{+}$and $K^{-}$mesons}

In heavy ion reactions, $K^{+}$and $K^{-}$mesons are created from elementary hadron-hadron collisions. Since only very energetic hadron-hadron collisions are able to produce a kaon (or antikaon), one usually assumes that no coherence exists between the different collisions

producing kaons. At SIS energies the major processes responsible for kaon and antikaon production are baryon-baryon collisions

$$
\begin{gathered}
B+B \rightarrow B+Y+K^{+} \\
B+B \rightarrow B+B+K^{+}\left(K^{0}\right)+K^{-}
\end{gathered}
$$

and pion-baryon collisions

$$
\begin{gathered}
\pi+B \rightarrow Y+K^{+} \\
\pi+B \rightarrow B+K^{+}\left(K^{0}\right)+K^{-} .
\end{gathered}
$$

Here B stands for a nucleon or nucleonic resonance while $\mathrm{Y}$ denotes a hyperon $(\Sigma$ or $\Lambda$ ). At SIS energies the relevant resonances are mainly $\Delta$ and $N^{*}$ resonances. If one goes to higher incident energies, e.g. to AGS energies ( about $10 \mathrm{GeV} /$ nucleon ), one must take into account contributions of heavier mesons and resonances [34]. For the baryon-baryon channels leading 
to $K^{+}$or $K^{-}$mesons, we have adopted theoretical cross sections based on the one-bosonexchange model. These cross sections agree well with experiments [36]. The $K^{+}$production cross section from the pion-induced channel has been evaluated within a resonance model [37, and a good agreement has been found between theory and data. The $K^{-}$production cross section from pion-baryon collisions is a parametrization of the experimental data [36]. In a previous study, it was shown that at SIS energies pion-baryon reactions can produce even more $K^{+}$mesons than baryon-baryon reactions [38. With an increasing number of participating nucleons, the $K^{+}$multiplicity originating from the $\pi$-induced channel increases much faster than that from the baryon-baryon channel. Hence this channel is essential in order to reproduce the mass dependence of the $K^{+}$production observed in experiments [39]. Furthermore, the pion-baryon reaction is important if one wants to understand the experimental observation of a non-isotropic $K^{+}$angular distribution in symmetric heavy ion reactions at subthreshold beam energies [40]. This is due to the fact that the pion-baryon reaction of Eq.(3) has a substantial P-wave component, which results in a large anisotropy. The P-wave contribution turned out to be essential for the understanding of the observed $K^{+}$angular distribution [41].

\section{Kaon reabsorption and rescattering}

Once produced a $K^{+}$meson can be hardly absorbed inside the medium because of strangeness conservation. However, a $K^{-}$meson can be easily destroyed through the reaction $K^{-}+\mathrm{N} \rightarrow \pi+\mathrm{Y}$. The inverse process also contributes partially to the $K^{-}$production. We used in our calculation a parametrization of the experimental cross section for these reactions [36]. The $K^{-}$absorption is rather strong. $\sigma_{K^{-} N \rightarrow \pi Y}$ is about $50 \mathrm{mb}$ at a laboratory $K^{-}$ momentum of $0.2 \mathrm{GeV} / \mathrm{c}$ and increases rapidly with decreasing momenta.

Both $K^{+}$and $K^{-}$mesons may scatter elastically with nucleons as propagating through the nuclear medium. The $K^{+}$-nucleon interaction is relatively weak $\left(\sigma_{K N} \sim 10 \mathrm{mb}\right)$ 42. 
However, in the initial stage of the nucleus-nucleus collisions at SIS energies, one can expect nuclear matter densites up to three times saturation density [33], which considerably shortens

the mean free path of the kaons $\left(\lambda=(\sigma \cdot \rho)^{-1}\right)$. This may lead to observable effects on the kaon spectra 41,43. Compared to $K^{+}$mesons, $K^{-}$mesons have a much larger elastic cross section, which is about $50 \mathrm{mb}$ at a laboratory $K^{-}$momentum of $0.2 \mathrm{GeV} / \mathrm{c}$ and becomes even larger with decreasing momenta. For the elastic $K^{+}$and $K^{-}$cross sections we use the parametrization given in Ref. [36].

\section{Kaon Coulomb interaction}

In Ref. [18] it was shown that the Coulomb interaction between the $K^{+}$mesons and the nuclear medium has a visible effect on the kaon dynamics in heavy systems like $\mathrm{Au}+\mathrm{Au}$. Thus the Coulomb interaction of both the $K^{+}$and $K^{-}$mesons is taken into account. The Coulomb field acting on the kaons is generated by protons, charged resonances and pions. We should notice that the multiplicity of negative charged pions has been observed to exceed greatly the value for positive charged pions in large systems such as $\mathrm{Au}+\mathrm{Au}$ [22, 32, 33]. This leads to a non-vanishing contribution of the pions to the charge density. Thus the total Coulomb interaction is given by the sum over all charged particles, i.e.

$$
U_{i_{k}}^{C o u}=\sum_{i_{n}} \frac{Z_{i_{k}} Z_{i_{n}} e^{2}}{\left|\vec{r}_{i_{k}}-\vec{r}_{i_{n}}\right|}+\sum_{i_{r}} \frac{Z_{i_{k}} Z_{i_{r}} e^{2}}{\left|\vec{r}_{i_{k}}-\vec{r}_{i_{r}}\right|}+\sum_{i_{\pi}} \frac{Z_{i_{k}} Z_{i_{\pi}} e^{2}}{\left|\vec{r}_{i_{k}}-\vec{r}_{i_{\pi}}\right|},
$$

where $i_{n}, i_{r}$ and $i_{\pi}$ denote nucleons, resonances $\left(\Delta\right.$ and $\left.N^{*}\right)$ and pions, respectively.

\section{E. Kaon mean field of the strong interaction}

The strong interaction of kaons with nuclear matter is expected to play also an important role in modifying the propagation of $K^{+}$and $K^{-}$mesons. Possible effects of the kaon-nuclear matter strong interaction have attracted special interest [17, 19,35,36]. The well-established formalism for studying kaon properties in nuclear matter is chiral perturbation theory. Based on a $\mathrm{SU}(3)_{L} \times \mathrm{SU}(3)_{R}$ chiral Lagrangian Kaplan and Nelson [44] constructed an effective 
meson-baryon Lagrangian, whose coefficients had been determined from experimental measurements, however, still with an uncertainty of about 30\%. Applying this Lagrangian to the $\mathrm{KN}$ interaction on the mean field level, one can obtain the following dispersion relation for a kaon or antikaon in nuclear matter [17]

$$
\begin{aligned}
& \omega_{K^{+}}=\sqrt{\vec{p}^{2}+m_{K}^{2}\left[1-\frac{\Sigma_{K N}}{f_{\pi}^{2} m_{K}^{2}} \rho_{S}+\left(\frac{3 \rho_{B}}{8 f_{\pi}^{2} m_{K}}\right)^{2}\right]}+\frac{3}{8} \frac{\rho_{B}}{f_{\pi}^{2}}, \\
& \omega_{K^{-}}=\sqrt{\vec{p}^{2}+m_{K}^{2}\left[1-\frac{\Sigma_{K N}}{f_{\pi}^{2} m_{K}^{2}} \rho_{S}+\left(\frac{3 \rho_{B}}{8 f_{\pi}^{2} m_{K}}\right)^{2}\right]}-\frac{3}{8} \frac{\rho_{B}}{f_{\pi}^{2}},
\end{aligned}
$$

where $m_{K}$ denotes the bare kaon mass, and $f_{\pi} \approx 93 \mathrm{MeV}$ is the pion decay constant. $\rho_{B}$ and $\rho_{S}$ are the baryon density and the scalar density, respectively. The kaon-nucleon sigma term $\Sigma_{K N}$ depends on the nucleon strangeness content and the strange quark mass. A value of $\Sigma_{K N}=350 \mathrm{MeV}$ has been used in the literature 17,36], however, recent lattice QCD calculations imply a higher value of $\Sigma_{K N}=450 \pm 30 \mathrm{MeV}$ [45]. While the $\Sigma_{K N}$ term provides a scalar attraction for both $K^{+}$and $K^{-}$mesons, the term proportional to $\rho_{B}$ gives rise to a vector potential, which is repulsive for $K^{+}$mesons and attractive for $K^{-}$mesons due to the G-parity transformation. The expressions Eq.(6) and Eq.(7) for the in-medium kaon potentials include only the S-wave interaction. There is also a P-wave contribution of kaons with the nuclear medium by hyperon-nucleon hole excitations. This $\mathrm{P}$-wave interaction changes signs from $K^{+}$to $K^{-}$mesons and is in its absolute value about twice as large for $K^{-}$than for $K^{+}$mesons. This P-wave interaction has till now been neglected in all previous works on kaons in nuclei. We will neglect it here also. But future investigations should study, if it is important. The strong potential for a kaon is in this approach defined as 46]

$$
U_{K}^{S t r}=\omega_{K}-\sqrt{m_{K}^{2}+\vec{p}^{2}}
$$

Considering the mean field of the strong interaction and the Coulomb field, the total Hamiltonian for the kaons reads

$$
H_{k}=\sum_{i_{k}}\left(\sqrt{m_{K}^{2}+\vec{P}_{i_{k}}^{2}}+U_{i_{k}}^{\text {Cou }}+U_{i_{k}}^{S t r}\right)
$$


There exist experimental constraints on the kaon mean field. As indicated above, the $K^{+} \mathrm{N}$ interaction is relatively weak compared to other hadron-nucleon interactions. Therefore, the impulse approximation should be justified to determine the in-medium $K^{+}$potential at low densities from experimental free KN scattering data. This yields a repulsive $K^{+}$potential of about $30 \mathrm{MeV}$ at saturation density $\left(\rho_{0}=0.16 \mathrm{fm}^{-3}\right)$, if one adopts an isospin averaged free KN scattering length $\bar{a}_{K N}=-0.255 \mathrm{fm}$ 477. On the other hand, experiments of kaonic atoms can provide direct information on the $K^{-}$mean field around $\rho_{0}$. An attractive $K^{-}$-nucleus potential of $U_{K^{-}}=-200 \pm 20 \mathrm{MeV}$ had been found in ${ }^{56} \mathrm{Ni}$ 48. With $\Sigma_{K N}=$ $350 \mathrm{MeV}$, one obtains from Eq.6-8 a $K^{+}$potential of about $+7 \mathrm{MeV}$ and a $K^{-}$one of -100 $\mathrm{MeV}$ at $\rho_{0}$. Both values are quite different from their respective empirical findings. High order corrections and $\mathrm{P}$-wave contributions to the mean field approximation may be responsible for this discrepancy. In Ref. 45,49, it was argued that the so-called range term, which is of the same order in chiral perturbation theory as the $\Sigma_{K N}$ term, has also contributions. In addition, the presence of the nuclear medium can reduce the pion decay constant $f_{\pi}$ by decreasing the quark condensates. Taking the range term and the in-medium reduction of $f_{\pi}$ into account, one obtains at $\rho_{0}$ about $+28 \mathrm{MeV}$ for the $K^{+}$and $-175 \mathrm{MeV}$ for the $K^{-}$ potential with a value of $\Sigma_{K N}=450 \mathrm{MeV}$. Thus the strength of the potentials is now roughly consistent with the respective empirical values.

The $K^{-}$-nuclear matter interaction is complicated by the $\Lambda(1405)$ resonance in the $K^{-}$nucleon channel, which is commonly considered to be an unstable $\bar{K} \mathrm{~N} \mathrm{I}=0$ bound state about $27 \mathrm{MeV}$ below the $K^{-}$p threshold. This state also appears as a resonance in the $\pi \Sigma$ channel. The $\Lambda(1405)$ is responsible for the repulsive isospin-averaged $\bar{K} \mathrm{~N}$ scattering length in free space 48]. Since the $\bar{K} \mathrm{~N}$ interaction is strong, the impulse approximation should not be good. A coupled channel description of the $\bar{K} \mathrm{~N}$ and $\pi$-hyperon channels is necessary. Such studies including the $\Lambda(1405)$ have been performed by Weise et al. 51,52. They show that the $\Lambda(1405)$ state dissolves in a nuclear environment due to Pauli blocking. Therefore, although the $\Lambda(1405)$ dominates the threshold $\bar{K} \mathrm{~N}$ dynamics at very low densities $(\rho<$ 
$\left.0.3 \rho_{0}\right)$, it is of minor relevance for the $K^{-}$-nuclear matter interaction at densities $\rho>\rho_{0}$. These calculations [51,52 have been performed with free kaons. A selfconsistent inclusion of the kaon-nucleus potential (in medium effects) may change these conclusions and should in the future be considered.

For the $K^{+}$mesons, we use the potential suggested in Ref. [45,49], since it agrees with the impulse approximation. For $K^{-}$mesons, a potential of $-200 \mathrm{MeV}$ at $\rho_{0}$ seems to be too deep to describe the experimental data of $K^{-}$production from heavy ion reactions [36,50. Thus one usually uses a $K^{-}$potential of about $-100 \mathrm{MeV}$ at normal nuclear matter density [36, 50]. We use a $K^{-}$potential derived from Eq.7-8 with $\Sigma_{K N}=350 \mathrm{MeV}$. The additional corrections from $\mathrm{P}$-wave contributions, the range term and the in-medium modification of the pion decay constant are thereby neglected. Such a $K^{-}$potential is more or less the same as used by other authors [36,50], and agrees reasonably well with coupled channel studies [51,52]. We will show later that the conclusions of the present work do not depend on the particular choice of the $K^{-}$potential. However, there seem to exist some discrepancies between the $K^{-}$potential extracted from the kaonic atoms and the one required for understanding $K^{-}$production from heavy ion reactions [36.50]. Future studies should shed light on this question. In Fig. 1 the corresponding potentials for $K^{+}$and $K^{-}$mesons used in the present work are shown for zero momentum. We note that the scalar density $\rho_{S}$ is model dependent. We followed a method used in the RBUU ( Relativistic Boltzmann-Uehling-Uhlenbeck ) model to calculate $\rho_{S}$ as a function of $\rho_{B}$ [53].

The kaon production thresholds might also be changed in the nuclear environment by the strong mean field $U^{S t r}$. ( We note that the Coulomb field $U^{\text {Cou }}$ has no net effect on the threshold due to charge conservation. ) The KaoS Collaboration [35] has observed an enhanced $K^{-}$production in nucleus-nucleus collisions as compared to free nucleon-nucleon collisions, which could be attributed to an attractive $K^{-}$mean field. Thus, we have taken into account the reduction of the $K^{-}$production threshold in a way similar as in Ref. [36]. 
The $K^{+}$mean field is much weaker than the $K^{-}$one. About medium modifications of the $K^{+}$production threshold exist different opinions in the literature 36,45,54. If such an effect exists, it should mainly affect the multiplicity of the kaons, rather than their collective motion. Therefore, we have not included any medium modification of the $K^{+}$production threshold in the present work, since the $K^{+}$mean field is much weaker than that for the $K^{-}$mesons. One can expect that the threshold is also much less affected.

The QMD model described above has been shown to be able to reproduce very well the experimental measurements of the multiplicities and transverse flow of nucleons, pions and kaons [18, 38,41,33]. In this work, we will apply the same model to other kaon collective flows (e.g. radial kaon flow).

\section{III. $K^{+}$RADIAL FLOW}

\section{A. Transverse mass spectra}

One way to obtain information on the collective motion in heavy ion reactions is to investigate particle multiplicities as a function of the transverse mass $\left(m_{t}=\sqrt{m_{0}^{2}+P_{T}^{2}}\right)$. In Fig. 2 we show the transverse mass spectrum of $K^{+}$mesons emitted at midrapidity ( $\left.-0.4<y_{c . m .} / y_{\text {proj }}<0.4\right)$ in the reaction $\mathrm{Au}+\mathrm{Au}$ at an incident energy of $1 \mathrm{GeV} /$ nucleon and an impact parameter of 3 and $5 \mathrm{fm}$, respectively. A striking finding of the present work is that the kaon spectrum exhibits as a function of the transverse mass a "shoulder-arm" shape, which deviates from a pure thermal picture. This becomes evident by comparing the kaon spectrum with a Boltzmann fit to the high energy part of the spectrum ( also shown in Fig. 2 ). In a pure thermal picture the kaons are produced from an equilibrated thermal source. The transverse mass spectra should thus satisfy a Boltzmann distribution, which is more or less a straight line if plotted logarithmically. A similar deviation from the thermal behavior had already been found for nucleons and composite particles ( deuterons, heliums etc. ), which was explained by introducing a collective expansion of the fireball, i.e. the 
nuclear matter radial flow [55 57. This expanding source picture has also been successfully applied to pion and kaon spectra at AGS energies ( about $10 \mathrm{GeV} /$ nucleon ) [58]. This seems to be reasonable since at such high beam energies hadron-hadron collisions occurring in the late expansion phase of the reaction are still energetic enough for kaon production. The kaons produced in the expanding nuclear matter should reflect a collective component transfered from the nucleons to kaons. However, it is not clear if this mechanism still works at subthreshold energies.

In Fig. 3 calculations are presented where the $K^{+}$potential $U^{S t r}$ and the Coulomb interaction $U^{C o u}$ have been dropped. Now one sees only effects of the hadron-hadron collisions, which produce the kaons. ( The $K^{+}$-nucleon elastic scattering is also included in the simplified calculations, however, its influence is fairly weak. ) The resultant kaon transverse mass spectrum with the same rapidity cut as in Fig. 2 is in exact agreement with a Boltzmann distribution. Thus, we can deduce that the "shoulder-arm" structure observed in Fig. 2 is caused by the kaon mean field and the Coulomb interaction, rather than by a collective expansion of the kaon sources. As pointed out in Ref. [18], the mean field and not the Coulomb interaction plays the dominant role in modifying the kaon motion. The fact that the potential and not an expanding $K^{+}$source is responsible for the "shoulder-arm" structure can be connected with the subthreshold beam energy. Only the hadron-hadron collisions during the early compression phase of the reaction have enough energy to produce a kaon. As the fireball starts to expand coherently in a later stage, the kaon production ceases to stop since the energetic collisions have been exhausted. Consequently, at a subthreshold beam energy no "shoulder-arm" spectrum for the midrapidity kaons can arise from the velocities of the sources. On the other hand, the $K^{+}$mean field is repulsive with a value of about $28 \mathrm{MeV}$ at saturation density $\left(\rho_{0}\right)$ and increases with the density. The kaons will thus inevitably experience an acceleration due to the repulsive potential as they propagate outwards from the overlap region. This may give rise to a collective motion of the kaons in the radial direction, which can lead to a deviation from the thermal picture. Therefore, 
the observed "shoulder-arm" structure in the kaon spectrum appears to be a signature of a genuine collective motion of the kaons, rather than only a result of the nuclear matter radial flow.

\section{B. Radial flow}

In order to quantitatively extract the collective component from the kaon spectrum, we fit the QMD results by incorporating a common radial velocity of the $K^{+}$mesons to the standard Boltzmann distribution. The corresponding distribution reads

$$
\frac{d^{3} N}{d \phi d y m_{t} d m_{t}} \sim e^{-\left(\frac{\gamma E}{T}-\alpha\right)}\left\{\gamma^{2} E-\gamma \alpha T\left(\frac{E^{2}}{p^{2}}+1\right)+(\alpha T)^{2} \frac{E^{2}}{p^{2}}\right\} \frac{\sqrt{(\gamma E-\alpha T)^{2}-m^{2}}}{p}
$$

where $E=m_{t} \cosh y, p=\sqrt{p_{t}^{2}+m_{t}^{2} \sinh ^{2} y}, \alpha=\gamma \beta p / T, \gamma=\left(1-\beta^{2}\right)^{-1 / 2}$. $\mathrm{T}$ is the temperature of the thermal source, while the parameter $\beta=v / \mathrm{c}$ is a common radial velocity of the kaons. Fig. 2 shows that Eq.(10) gives a good fit to the QMD results. The fit yields for $\mathrm{b}=5 \mathrm{fm}$ a temperature of $\mathrm{T}=60 \mathrm{MeV}$ and a common velocity of $\beta=0.1$. A slightly higher temperature $(\mathrm{T}=62 \mathrm{MeV})$ and larger common velocity $(\beta=0.11)$ are obtained for more central collisions ( $\mathrm{b}=3 \mathrm{fm}$ ). Are the $\mathrm{T}$ and $\beta$ derived from the fit a good measure of the thermal and collective motion of the kaons? As we mentioned above, the thermal feature of the kaon spectra described by T reflects the temperature of the kaon sources, while the collective component - given by $\beta$ - is due to the acceleration of the kaons by the mean field. Thus the extracted temperature describes the thermal motion without the influence of the kaon potential ( see Fig. 3 ). On the other hand, the collective motion $\beta$ can be quantitatively described by the enhancement of the kaon momentum by the repulsive $K^{+}$ potential. The simulations shown in Fig. 3 give temperatures of $\mathrm{T}=64 \mathrm{MeV}$ for $\mathrm{b}=5 \mathrm{fm}$ and $\mathrm{T}=66 \mathrm{MeV}$ for $\mathrm{b}=3 \mathrm{fm}$, respectively. They are quite comparable to the temperatures T extracted from the fit to the full calculations (Fig. 2). The average transverse mass of the kaons is increasing from $\left\langle m_{t}\right\rangle=566 \mathrm{MeV}$ to $594 \mathrm{MeV}$ for $\mathrm{b}=5 \mathrm{fm}$ as one changes from the simulations without to those including the kaon potential. For $\mathrm{b}=3 \mathrm{fm}$ it increases 
from $\left\langle m_{t}\right\rangle=571 \mathrm{MeV}$ to $600 \mathrm{MeV}$. ( We note that the Coulomb interaction contributes less than $30 \%$ to the enhancements. ) This corresponds to a common radial velocity boost of the kaons by $\beta=0.102$ and $\beta=0.113$ for $\mathrm{b}=5 \mathrm{fm}$ and $3 \mathrm{fm}$, respectively. The two values are very close to the $\beta$ parameters obtained by the fit. This indicates that it is possible to separate the thermal and the collective components of the kaon spectrum by the present analysis.

At an incident energy of $1 \mathrm{GeV} /$ nucleon, the energy available for kaon production is quite limited. Consequently, the kaons have also a limited thermal motion. The average radial velocity of the kaons due to thermal motion is about 0.488 ( in unit of the light velocity c ) for $\mathrm{Au}+\mathrm{Au}$, while the flow velocity is about $\beta=0.1$ as mentioned above. The collective motion is more than $20 \%$ of the thermal motion, so that it is not obscured by the latter, and can be observed as a clear signal.

The radial collective motion of the $K^{+}$mesons may provide a new opportunity for investigation of the kaon mean field in nuclear matter. As stronger the kaon potential, as more significant is the radial kaon flow. Thus the kaon radial flow can be used as a meter to determine the kaon in-medium potential. We note that an analysis of the transverse momentum of the kaons as a function of the rapidity (so-called kaon transverse flow) might give also information of the kaon potential. Without the kaon mean field, the transverse kaon flow follows the flow patterns of the baryons, and exhibits a S-shape. This has been found in both RBUU calculations [17] and previous studies using the QMD model [18]. The S shape disappears and a zero flow can be observed if one takes into account the kaon potential as found in Ref. 17 19.

\section{IV. $K^{-}$"VIRTUAL" RADIAL FLOW}

In contrast to the $K^{+}$mesons studied in the preceding section, many other hadrons such as $K^{-}, \Lambda, \Sigma$ etc. are supposed to feel an attractive mean field in nuclear matter. In 
addition they may be strongly absorbed or scattered in the medium. If these hadrons can also develop a radial collective motion in heavy ion reactions is not clear. Therefore, it is of high interest to investigate the possible formation of radial flow of these hadrons. As a first example, we consider in the present work $K^{-}$mesons which are subjected to all the final-state interactions mentioned above.

In Fig. 4 the $K^{-}$transverse mass spectrum at midrapidity is shown for the $\mathrm{Au}+\mathrm{Au}$ reaction at a single impact parameter $\mathrm{b}=5 \mathrm{fm}$ and for the $\mathrm{Ni}+\mathrm{Ni}$ reaction by integrating over impact parameters $0<\mathrm{b}<4 \mathrm{fm}$. The considered beam energies $(1.8 \mathrm{GeV} /$ nucleon for the $\mathrm{Au}+\mathrm{Au}$ reaction and $1.93 \mathrm{GeV} /$ nucleon for the $\mathrm{Ni}+\mathrm{Ni}$ reaction ) are below the free NN $K^{-}$threshold. We analysed the $K^{-}$mesons from a rapidity interval $\left(-0.3<y_{c . m .} / y_{\text {proj }}\right.$ $<0.3$ ) normalized to projectile rapidity $y_{p r o j}$, which looks narrower than the one for $K^{+}$ mesons $\left(-0.4<y_{c . m .} / y_{\text {proj }}<0.4\right)$. In fact, the size of the intervals in units of rapidity is very close to each other, since we consider for the $K^{-}$production higher beam energies than for the $K^{+}$production due to the different thresholds ( $2.5 \mathrm{GeV}$ compared to 1.58 $\mathrm{GeV}$ ). The rapidity of the $K^{+}$or $K^{-}$mesons is explicitly included in the equations fitting the kaon spectra ( see Eq.10 for the $K^{+}$case and Eq.11 to appear later in this section for the $K^{-}$case ). Therefore, small differences between the rapidity intervals for the $K^{+}$ and $K^{-}$mesons should not influence the conclusions of this study. Also shown in Fig.4 is a Boltzmann fit to the high energy part of the spectrum $\left(m_{T}-m_{K^{-}}>0.1 \mathrm{GeV}\right)$. One can clearly observe a "concave" structure in the $K^{-}$spectrum again deviating from a thermal Boltzmann distribution. This structure is, however, completely different from the "shoulder-arm" shape found for $K^{+}$mesons.

The "concave" structure is a consequence of the attractive $K^{-}$mean field. Without the mean field this structure vanishes. ( The present work also includes the Coulomb interaction of the $K^{-}$mesons. However, the Coulomb effects are found to be negligible compared to those originating from the strong potential. ) Fig. 5 demonstrates that the $K^{-}$spectra 
obtained without the mean field and the Coulomb interaction ( but including rescattering and reabsorption ) are in good agreement with a Boltzmann distribution. At a subthreshold beam energy hadron-hadron collisions, i.e. sources for the $K^{-}$mesons, can not induce this "concave" structure since the $K^{-}$'s are mainly produced before the fireball starts to expand. We already discussed this point in Section III. It is important to realize that also reabsorption and rescattering do not lead to the observed "concave" structure. The transverse mass spectrum of the midrapidity $K^{-}$mesons is determined primarily by the interaction of the $K^{-}$mesons with the fireball. If one neglects all the final-state interactions, the primordial $K^{-}$spectra should satisfy according to Fermi's argument 28] a pure Boltzmann distribution. On the other side, the fireball baryons show predominantly a single-temperature structure before the formation of the nuclear matter radial flow. We know that both rescattering and reabsorption are in nature stochastic processes which tend to thermalize a narrow distribution. One can hardly expect that a stochastic interaction between two thermal distributions can change the thermal distributions to a two-temperature structure. Thus one observes in Fig. 5 a Boltzmann distribution of the $K^{-}$mesons even though rescattering and reabsorption have been included. However, we should note that rescattering and reabsorption of hadrons by spectators can induce non-thermal features since the spectators are collectively deflected sidewards in the reaction plan ( nuclear matter bounce-off ). Absorption of pions by the spectator baryons is responsible for a pion transverse flow which is anticorrelated to the nucleon flow [14 16]. Rescattering and reabsorption by the spectators can also give rise to an azimuthal anisotropy in the hadron emission (Squeeze-out) [20 22].

In Section III we have shown that the $K^{+}$radial flow can be described very well by boosting a Boltzmann distribution with a common $K^{+}$radial velocity directed away from the fireball as a result of the repulsive $K^{+}$mean field. Here we try to understand the $K^{-}$spectra with a similar boost, however, now with a common $K^{-}$radial velocity tending towards the center of the fireball since the $K^{-}$mesons feel an attractive mean field. In analogy to Eq.(10) the $K^{-}$distribution reads 


$$
\frac{d^{3} N}{d \phi d y m_{t} d m_{t}} \sim e^{-\left(\frac{\gamma E}{T}+\alpha\right)}\left\{\gamma^{2} E+\gamma \alpha T\left(\frac{E^{2}}{p^{2}}+1\right)+(\alpha T)^{2} \frac{E^{2}}{p^{2}}\right\} \frac{\sqrt{(\gamma E+\alpha T)^{2}-m^{2}}}{p} .
$$

$\mathrm{T}$ is the primordial $K^{-}$temperature, $\beta$ is the common $K^{-}$velocity. Fig. 4 shows that Eq.(11) provides a good fit to the $K^{-}$spectrum obtained from the full calculation. The adjusted temperature parameters $(\mathrm{T}=83 \mathrm{MeV}$ and $76 \mathrm{MeV}$ for $\mathrm{Au}+\mathrm{Au}$ and $\mathrm{Ni}+\mathrm{Ni}$, respectively ) are again close to these values deduced from a Boltzmann fit to the QMD results without the mean field ( $\mathrm{T}=87 \mathrm{MeV}$ and $82 \mathrm{MeV}$ for the two reactions, respectively ). This indicates that the fitting parameter $\mathrm{T}$ in Eq.(11) is a good measure for the thermal motion of the $K^{-}$mesons. The average transverse mass of the $K^{-}$mesons is found to be reduced by the mean field from $\left\langle m_{T}\right\rangle=596 \mathrm{MeV}$ to $575 \mathrm{MeV}$ for $\mathrm{Au}+\mathrm{Au}$, and from $585 \mathrm{MeV}$ to $569 \mathrm{MeV}$ for $\mathrm{Ni}+\mathrm{Ni}$, which correspond to a radial decelerating boost of $\beta=$ 0.083 and 0.067 , respectively. Both $\beta$ values agree with that from the fit ( $\beta=0.085$ for $\mathrm{Au}$ $+\mathrm{Au}$ and 0.066 for $\mathrm{Ni}+\mathrm{Ni}$ ). Therefore, the non-thermal feature of the $K^{-}$mesons can be described well by a radial velocity $\beta$ extracted from the fit.

The deviation of the $K^{-}$transverse mass spectra from a pure thermal distribution can therefore be understood in terms of a common $K^{-}$radial collective motion, which arises from the deceleration by the attractive mean field. We call this type of collective motion "virtual" flow in order to distinguish it from the $K^{+}$radial flow driven by a repulsive mean field. Such a virtual flow is characterized by a "concave" structure in the transverse mass spectrum, while a radial flow shows up in a "shoulder-arm" shape of the spectrum.

Rescattering and reabsorption alone can not induce the "concave" feature in the $K^{-}$ spectra. However, a stochastic process may interfere with a collective motion as mentioned above. Reabsorption plays for $K^{-}$mesons a larger role than for $K^{+}$mesons. $K^{-}$rescattering on the fireball baryons may increase the energy of $K^{-}$mesons. This will cancel partially the virtual flow caused by the mean field. Therefore, we observe in the present work a relative small reduction of the $K^{-}$transverse mass $\left(\Delta<m_{T}>=-21 \mathrm{MeV}\right.$ and $-16 \mathrm{MeV}$ for $\mathrm{Au}+$ $\mathrm{Au}$ and $\mathrm{Ni}+\mathrm{Ni}$, respectively. ) although we have adopted a mean field for the $K^{-}$-nuclear 
matter interaction of about $-100 \mathrm{MeV}$ at normal nuclear matter density.

A "concave" structure had been observed experimentally in the transverse mass spectra for $K^{+}$and $K^{-}$mesons in $\mathrm{Si}+\mathrm{Pb}$ at $14.6(\mathrm{GeV} / \mathrm{c}) /$ nucleon [59] and $\mathrm{Au}+\mathrm{Au}$ at 10.8 $(\mathrm{GeV} / \mathrm{c}) /$ nucleon [60] as well as for pions in many reactions and at various beam energies [61]. In all the cases the beam energies are much higher than the corresponding production thresholds for free NN collisions. Considerable numbers of kaons or pions are produced even after the fireball starts to expand. Thus the fireball expansion can independently cause nontrivial collective features in the particle spectra [57,58]. Resonance decay producing pions has also a significant contribution, which had been found to be able to induce a "concave" structure in pion transverse mass spectra [30,31,62. It is worth noting that some explanations for these observations have also used an attractive pion or kaon in-medium mean field [63]. However, it seems that there exist some differences between these observations and our present work where the subthreshold beam energy plays an essential role.

Finally we want to add some remarks concerning the kaon potentials used in this work. For the description of the $K^{+}$radial flow a potential has been used which agrees with the free KN scattering data. Based on the same potential, we were able to reproduce the experimental $K^{+}$transverse flow data in a previous work [18]. It is also unlikely for resonance decay to modify the "shoulder-arm" shape of the $K^{+}$spectra, since, at the incident energies studied here, very few Phi mesons and antibaryons can be created. The $K^{-}$potential applied is less attractive than that extracted from kaonic atoms. Since the deceleration of the $K^{-}$ mesons by the attractive mean field is responsible for the "concave structure" of the $K^{-}$ spectra, a more attractive potential should even favour a more pronounced "concave" shape. The $\Lambda(1405)$ might also play a role in leading to a "concave" structure of the $K^{-}$spectra just analogous to the role of the $\Delta$ resonances for pions. However, the formation of the $\Lambda(1405)$ is reduced at high densities. Since the $K^{-}$radial flow arises from interactions with the dense fireball, one can expect that the $\Lambda(1405)$ is only of minor importance. We also 
note that our treatment of the kaon dynamics is obviously non-covariant, since e.g. the space component of the vector part of kaon-nucleus potential is neglected as usual [64]. However, this treatment is sufficient for the present studies, since the relative motion between the fireball and the midrapidity kaons is quite small at the subthreshold beam energies. The present conclusions are not significantly affected by a full covariant treatment of the kaon dynamics.

\section{SUMMARY}

In this work, we have studied possible collective motion of $K^{+}$and $K^{-}$mesons emitted at midrapidity from heavy ion reactions at SIS energies (1-2 GeV/nucleon) by comparing with a thermal picture. The main findings are:

(1). A collective flow of $K^{+}$mesons in the radial direction should exist in central nucleusnucleus collisions, which can be identified by a characteristic "shoulder-arm" shape of the transverse mass spectrum of the midrapidity kaons. The $K^{+}$radial flow is caused by the repulsive $K^{+}$mean field.

(2). $K^{-}$mesons also exhibit a collective motion in the radial direction in heavy ion reactions at subthreshold beam energies even though they suffer strong reabsorption and rescattering in the nuclear medium. The collective motion leads to a characteristic "concave" structure in the transverse mass spectrum of $K^{-}$mesons emitted at midrapidity. This distinguishes the $K^{-}$collective motion from that of $K^{+}$mesons. The $K^{-}$collective motion is induced by an attractive mean field.

(3). Since the radial flow of both $K^{+}$and $K^{-}$mesons arises from their respective inmedium mean field, the radial flow may act as a novel tool for investigation of possible modifications of kaon properties in dense nuclear matter. 
[1] H.Stöcker, J.A.Maruhn and W.Greiner, Phys. Rev. Lett. 44, 725, (1980).

[2] H.A.Gustafsson et al., Phys. Rev. Lett. 52, 1590 (1984).

[3] P.Danielewicz and G.Odyniec, Phys. Lett. B157, 146 (1985).

[4] H.Stöcker, L.P.Csernai, G.Graebner, G.Buchwald, H.Kruse, R.Y.Cusson, J.A.Maruhn and W.Greiner, Phys. Rev. C25, 1873 (1982).

[5] H.H.Gutbrod, K.H.Kampert, B.W.Kolb, A.M.Poskanzer, H.G.Ritter and H.R.Schmidt, Phys. Lett. B216, 267 (1989).

[6] M.A.Lisa and the EOS Collaboration, Phys. Rev. Lett. 75, 2662 (1995).

[7] I.G.Bearden and the NA44 Collaboration, Phys. Rev. Lett. 78, 2080 (1997).

[8] E.Schnedermann and U.Heinz, Phys. Rev. Lett. 69, 2908 (1992).

[9] Q.Pan and P.Danielewicz, Phys. Rev. Lett. 70, 2062 (1993).

[10] S.A.Bass, C.Hartnack, H.Stöcker and W.Greiner, Z. Phys. A 352, 171 (1995).

[11] H.Sorge, Phys. Rev. Lett. 78, 2309 (1997).

[12] S.A.Voloshin, Phys. Rev. C55, R1630 (1997).

[13] J.Barrette and the E877 Collaboration, Phys. Rev. Lett. 73, 2523 (1994).

[14] B.A.Li and W.Bauer, Phys. Rev. C44, 2095 (1991).

[15] S.A.Bass, C.Hartnack, R.Mattiello, H.Stöcker and W.Greiner, Phys. Lett. B302, 381 (1993).

[16] J.C.Kintner et al., Phys. Rev. Lett. 78, 4165 (1997).

[17] G.Q.Li, C.M.Ko and B.A.Li, Phys. Rev. Lett. 74, 235 (1995).

[18] Z.S.Wang, Amand Faessler, C.Fuchs, V.S.Uma Maheswari and D.Kosov, Nucl. Phys. A628, 
$151(1998)$.

[19] J.L.Ritman and the FOPI Collaboration, Z. Phys. A352, 355 (1995).

[20] D.Brill and the KaoS Collaboration, Phys. Rev. Lett. 71, 336 (1993).

[21] L.B.Venema and the TAPS Collaboration, Phys. Rev. Lett. 71, 835 (1993).

[22] S.A.Bass, C.Hartnack, H.Stöcker and W.Greiner, Phys. Rev. C51, 3343 (1994).

[23] P.Senger and the KaoS Collaboration, International Workshop on Hadrons in Dense Matter, GSI, Darmstadt, 1996.

[24] A.Jahns, C.Spieles, H.Sorge, H.Stöcker and W.Greiner, Phys. Rev. Lett. 72, 3464 (1994).

[25] D.E.Kahana, D.Keane, Y.Pang, T.Schlagel and S.Wang, Phys. Rev. Lett. 74, 4404 (1995).

[26] G.Q.Li, C.M.Ko, Phys. Rev. C54, R2159 (1996); 1897 (1996).

[27] G.E.Brown and H.A.Bethe, Astrophys. J. 423, 659 (1994); and references therein.

[28] E.Fermi, Prog. Theor. Phys. 5, 570 (1950).

[29] J.Aichelin, Phys. Reports. 202, 233 (1991).

[30] B.Hong and the FOPI Collaboration, Phys. Lett. B407, 115 (1997).

[31] E.L.Hjort et al., Phys. Rev. Lett. 79, 4345 (1997).

[32] W.Benenson et al., Phys. Rev. Lett. 43, 683 (1979); G.Bertsch, Nature 283, 280 (1980); M.Gyulassy and S.K.Kauffmann, Nucl. Phys. A362, 503 (1981).

[33] V.S.Uma Maheswari, C.Fuchs, Amand Faessler, L.Sehn, D.Kosov and Z.S.Wang, Nucl. Phys. A628, 669 (1998); C.Fuchs, L.Sehn, E.Lehmann, J.Zipprich and Amand Faessler, Phys. Rev. C55, 411 (1997).

[34] H.Sorge, R.Mattiello, A.Jahns, H.Stöcker and W.Greiner, Phys. Lett. B271, 37 (1991).

[35] R.Barth and the KaoS Collaboration, Phys. Rev. Lett. 78, 4007 (1997). 
[36] W.Cassing, E.L.Bratkovskaya, U.Mosel, S.Teis and A.Sibirtsev, Nucl. Phys. A614, 415 (1997).

[37] K.Tsushima, S.W.Huang and Amand Faessler, Phys. Lett. B337, 245 (1994); K.Tsushima, S.W.Huang and Amand Faessler, J. Phys. G21, 33 (1995).

[38] C.Fuchs, Z.S.Wang, L.Sehn, Amand Faessler, V.S. Uma Maheswari and D. Kosov, Phys. Rev. C56, R606 (1997).

[39] D.Miskowiec and the KaoS Collaboration, Phys. Rev. Lett. 72, 3650 (1994).

[40] R.Elmer et al., Phys. Rev. Lett. 77, 4884 (1996).

[41] Z.S.Wang, Amand Faessler, C.Fuchs, V.S.Uma Maheswari and D.Kosov, Phys. Rev. Lett. 79, 4096 (1997).

[42] C.Dover and G.E.Walker, Phys. Reports 89, 1 (1982).

[43] W.Zwermann and B.Schürmann, Nucl. Phys. A423, 525 (1984); X.S.Fang, C.M.Ko and Y.M.Zheng, Nucl. Phys. A556, 499 (1993).

[44] D.B. Kaplan and A.E. Nelson, Phys. Lett. B173, 57 (1986).

[45] G.E.Brown and M.Rho, Phys. Reports. 269, 333 (1996), and references therein.

[46] E.Shuryak and V.Thorsson, Nucl. Phys. A536, 739 (1992).

[47] T.Barnes and E.S.Swanson, Phys. Rev. C49, 1166 (1994).

[48] C.J.Batty, E.Friedman and A.Gal, Phys. Reports 287, 385 (1997).

[49] G.E.Brown, C.M.Ko and G.Q.Li, nucl-th/9608039.

[50] G.Q.Li, C.-H.Lee and G.E.Brown, Phys. Rev. Lett. 79, 5214 (1997).

[51] N.Kaiser, P.B.Siegel and W.Weise, Nucl. Phys. A594, 325 (1995).

[52] T.Waas, N.Kaiser and W.Weise, Phys. Lett. B365, 12 (1996); Phys. Lett. B379, 34 (1996).

[53] K.Weber, B.Blättel, W.Cassing, H.-C. Dönges, V.Koch, A.Lang and U.Mosel, Nucl. Phys. 
A539, 713 (1992).

[54] X.S.Fang, C.M.Ko, G.Q.Li and Y.M.Zheng, Nucl. Phys. A575, 766 (1994).

[55] P.J.Siemens and J.O.Rasmussen, Phys. Rev. Lett. 42, 880 (1979).

[56] R.Mattiello, A.Jahns, H.Sorge, H.Stöcker and W.Greiner, Phys. Rev. Lett. 74, 2180 (1995).

[57] P.Danielewicz, Phys. Rev. C51, 716, (1995).

[58] K.S.Lee, U.Heinz and E.Schnedermann, Z. Phys. C48, 525 (1990).

[59] J.Stachel and the E814 Collaboration, Nucl. Phys. A566, 183c (1994).

[60] J.Barrette and the E877 Collaboration, Nucl. Phys. A590, 259c (1995).

[61] R.Brockmann et al., Phys. Rev. Lett. 53, 2012 (1984); H.Ströbele and the NA35 Collaboration, Z. Phys. C38, 89 (1988); I.G.Bearden and the NA44 Collaboration, Phys. Rev. Lett. 78, 2080 (1997).

[62] H.Sorge, H.Stöcker and W.Greiner, Ann. Phys. (NY) 192, 266 (1989); T.J.Schlagel, S.H.Kahana and Y.Pang, Phys. Rev. Lett. 69, 3290 (1992).

[63] L.Xiong, C.M.Ko and V.Koch, Phys. Rev. C47, 788 (1993); V.Koch, Phys. Lett. B351, 29 (1995).

[64] C.Fuchs, D.Kosov, Amand Faessler, Z.S.Wang and T.Waindzoch, nucl-th/9801048. 


\section{Figure Captions}

Fig. 1. The in-medium potentials of $K^{+}$and $K^{-}$mesons in nuclear matter used in this work.

Fig. 2. Transverse mass spectra of the $K^{+}$mesons emitted at midrapidity ( $\left.0.4<y_{\text {c.m. }} / y_{\text {proj }}<0.4\right)$. The histograms are results of the QMD simulations for $\mathrm{Au}+\mathrm{Au}$ reactions at $1 \mathrm{GeV} /$ nucleon and at two different impact parameters $\mathrm{b}=3,5 \mathrm{fm}$. The calculations are performed including the full in-medium kaon dynamics. The lines are fits to the transport calculations. In the upper panel the result of a pure thermal Boltzmann fit to the high energy part of the spectra $\left(m_{t}-m_{0}>0.15 \mathrm{GeV}\right)$ is shown. In the lower panel the fit according to Eq.(10) is presented where one assumes a $K^{+}$radial flow in addition to the thermal motion.

Fig. 3. Transverse mass spectra of the $K^{+}$mesons emitted at midrapidity ( $\left.0.4<y_{\text {c.m. }} / y_{\text {proj }}<0.4\right)$ for the same reactions as in Fig. 2. The histograms present results of the transport calculations without the $K^{+}$mean field and Coulomb potential. Now a pure thermal fit (lines ) is sufficient to reproduce the spectra.

Fig. 4. Transverse mass spectra of the $K^{-}$mesons emitted at midrapidity $(-0.3<$ $\left.Y_{\text {c.m. }} / Y_{\text {proj }}<0.3\right)$ in a semicentral $(\mathrm{b}=5 \mathrm{fm}) \mathrm{Au}+\mathrm{Au}$ reaction at $1.8 \mathrm{GeV} /$ nucleon and in a $\mathrm{Ni}+\mathrm{Ni}$ reaction at $1.93 \mathrm{GeV} /$ nucleon. The $\mathrm{Ni}+\mathrm{Ni}$ reaction is averaged over the impact parameter region $0<\mathrm{b}<4 \mathrm{fm}$. The histograms are the results of the transport calculations including the full $K^{-}$in-medium dynamics and the lines are the corresponding fits to the spectra. In the upper panel the result of a pure thermal Boltzmann fit to the high energy part of the spectra $\left(m_{t}-m_{0}>0.1 \mathrm{GeV}\right)$ is shown. In the lower panel the fit according to Eq.(11) is presented where one assumes a $K^{-}$"virtual" radial flow in addition to the thermal motion.

Fig. 5. Transverse mass spectra of the $K^{-}$mesons emitted at midrapidity $(-0.3<$ 
$\left.Y_{\text {c.m. }} / Y_{\text {proj }}<0.3\right)$ for the same reactions as in Fig. 4. The histograms present results of the transport calculations without the $K^{-}$mean field and Coulomb potential. Now a pure thermal fit (lines ) is sufficient to reproduce the spectra. 


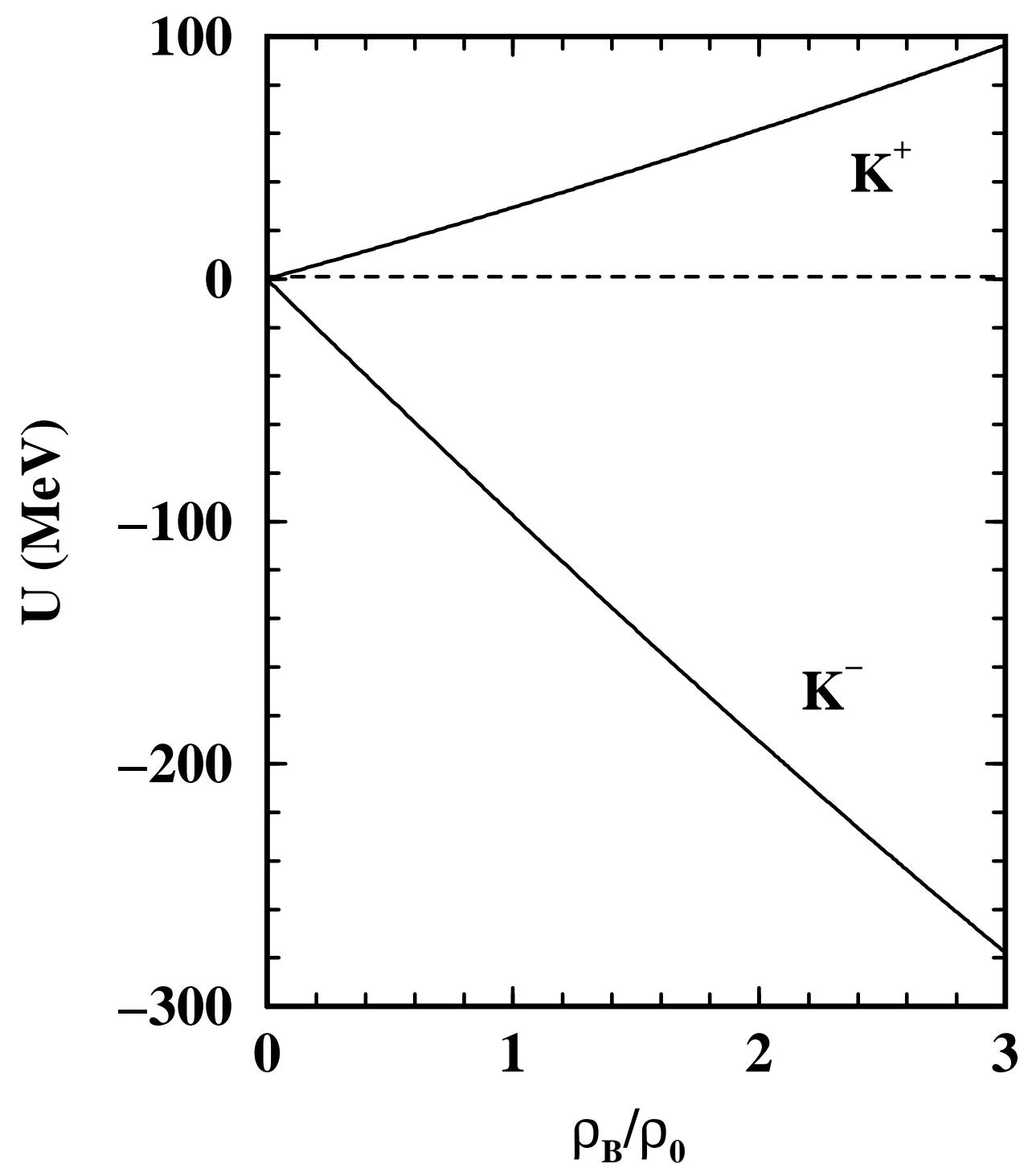

FIG. 1. 


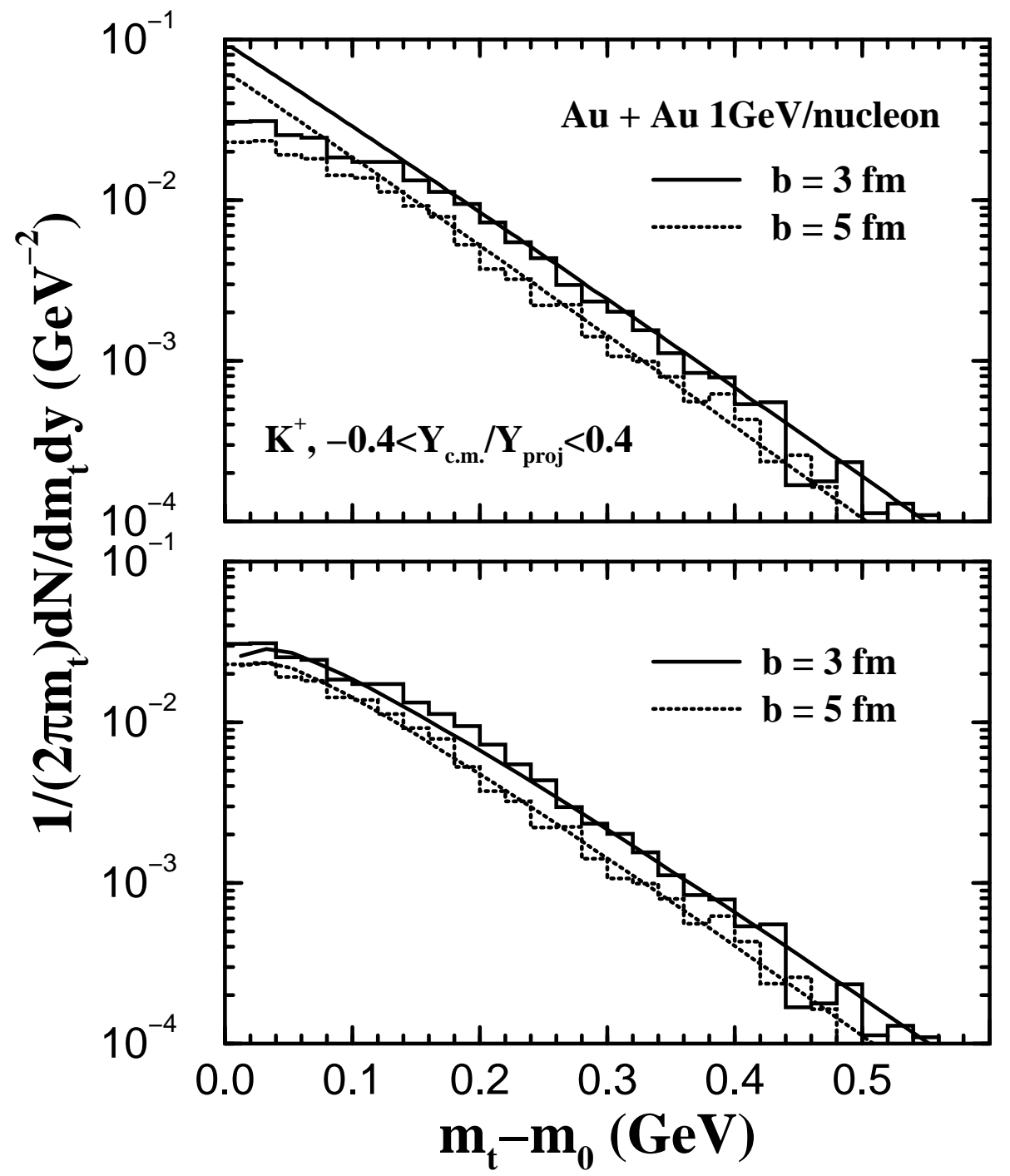

FIG. 2. 


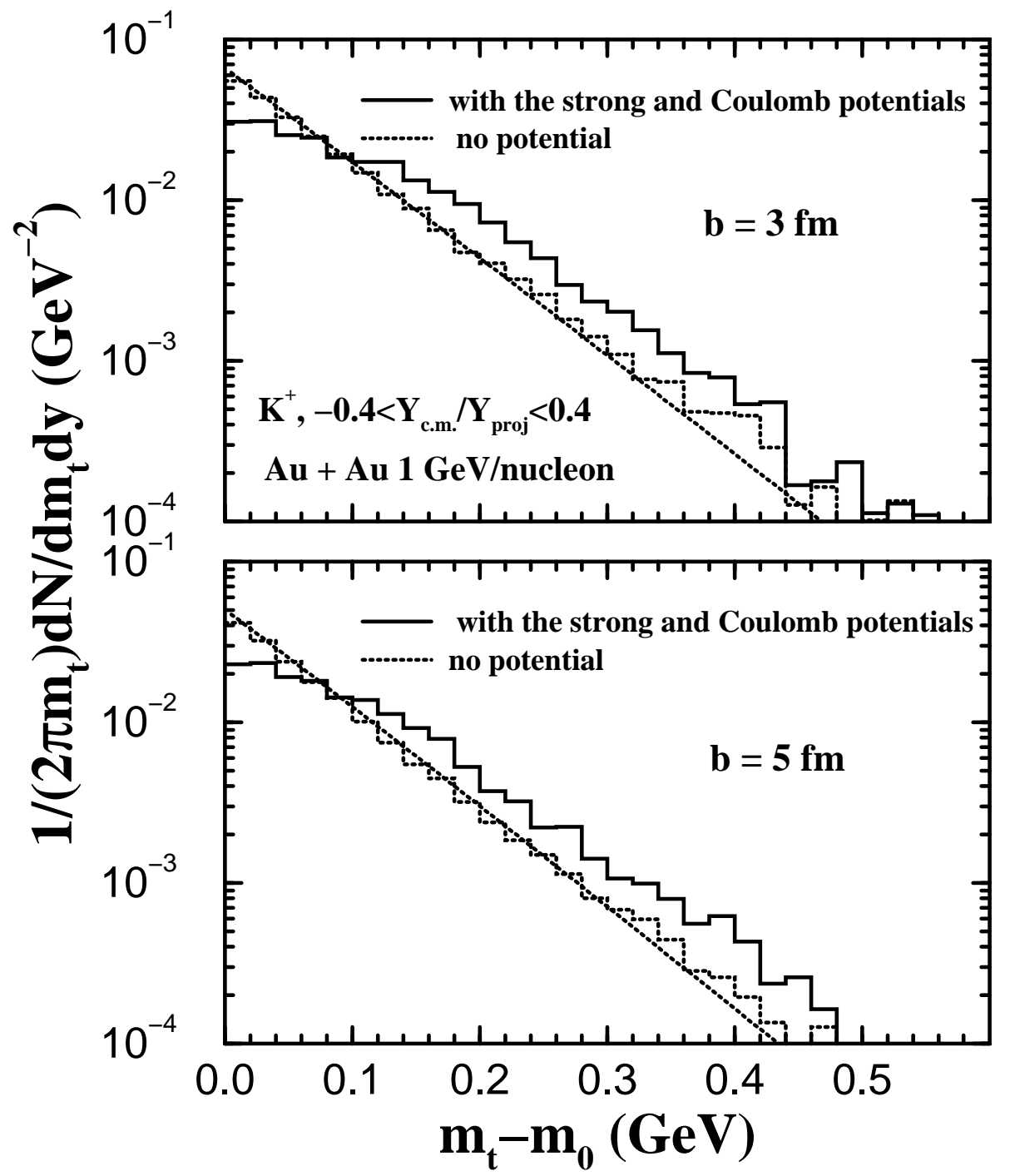

FIG. 3. 


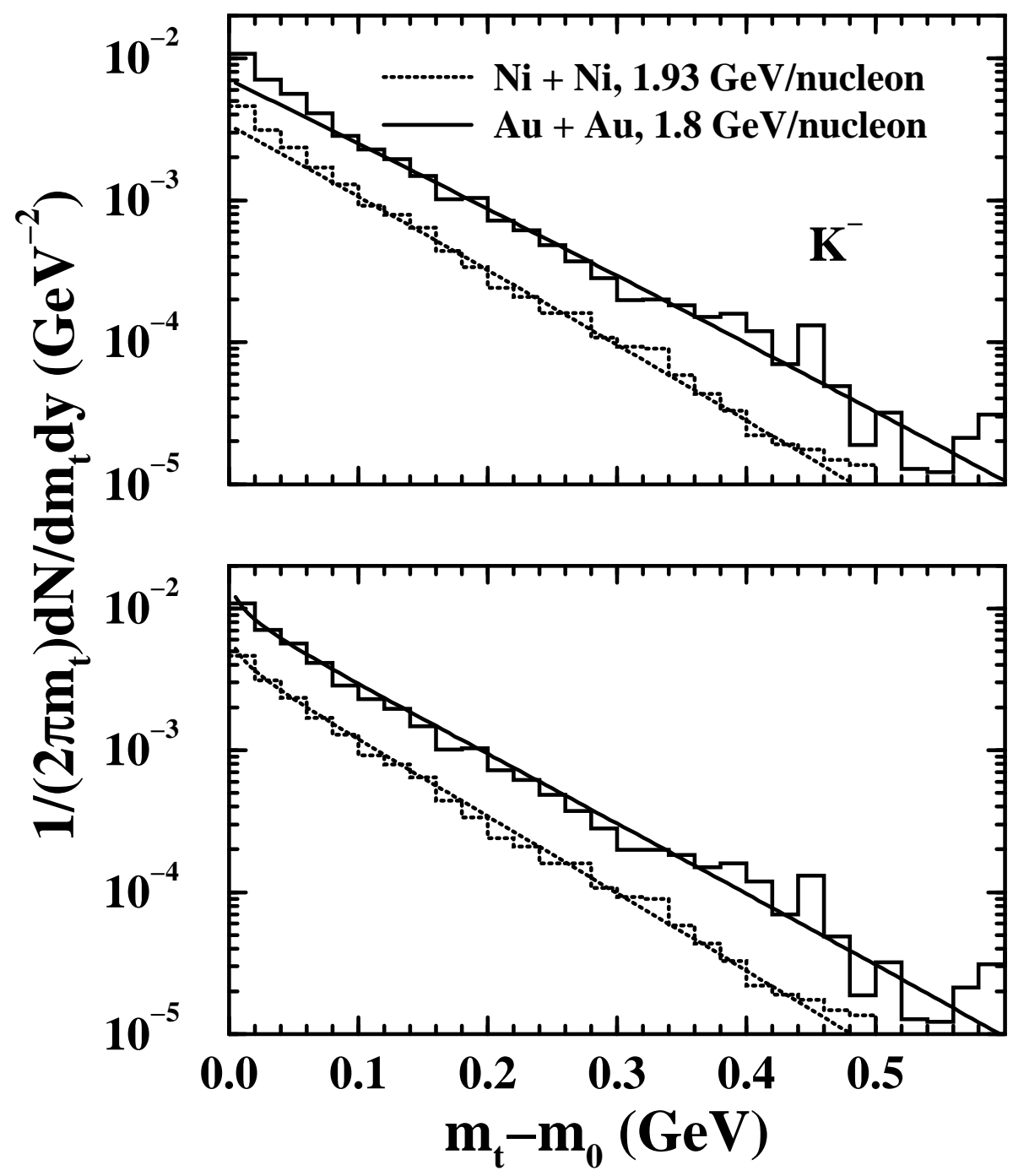

FIG. 4. 


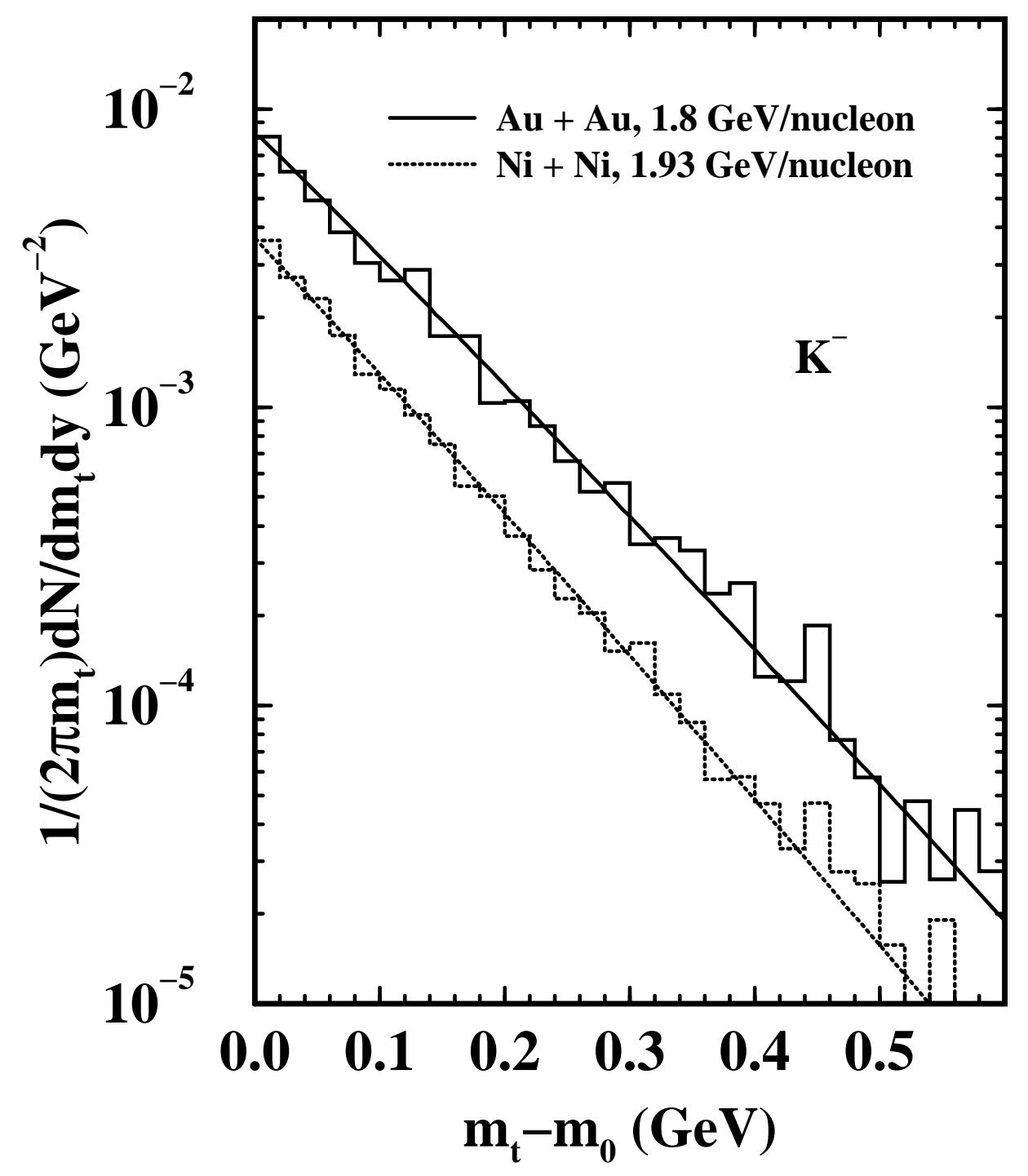

FIG. 5. 